\title{
Nonexistence of Some Quasi-Conformal Harmonic Diffeomorphisms
}

\author{
LEI NI
}

\section{Introduction}

The property of harmonic maps between complete Riemannian manifolds has been studied extensively by many authors (e.g., [C; Sh; T]). In the present paper we show some nonexistence results for quasi-conformal harmonic diffeomorphisms between complete Riemannian manifolds. In dimension 2, harmonic maps are closely related to the deformation theory of Riemann surfaces. One of the questions that arises naturally is: Are Riemann surfaces that are related by harmonic diffeomorphisms necessarily quasi-conformally related? See Schoen's article [S] for a general discussion on this subject, where other questions were also discussed. The result we show in this paper provides some partial answers to the high-dimensional generalization of this type of question. In particular we prove the following result, which can be thought of as a Liouville type theorem for harmonic diffeomorphisms.

THEOREM 1.1. Let $M^{n}$ be a complete manifold with Ricci $_{M} \geq 0$, and let $N^{n}$ be a simply connected manifold with nonpositive sectional curvature, where $n$ is the dimension of both manifolds. If there is a point $p \in M$ such that $\lim _{r \rightarrow \infty} V_{p}(r)=$ $o\left(r^{n}\right)$, then there is no quasi-conformal harmonic diffeomorphism from $M$ into $N$ with polynomial growth energy density.

It is not surprising that the growth rate of energy density plays a role here. For example, Wan proved [W] that a harmonic diffeomorphism between hyperbolic spaces of dimension 2 is quasi-conformal if and only if it has bounded energy density. The "only if" part of Wan's theorem was generalized to high dimension in [LTW]. Where it was proved that if the Ricci curvature of the domain manifold is bounded from below and if the first eigenvalue of the target manifold is positive, then any quasi-conformal harmonic diffeomorphism into the target manifold has bounded energy density. These results and some other related results in [HTTW] all indicate that the growth condition on the energy density is a natural assumption and is closely related to the study of quasi-conformal diffeomorphisms. On the other hand, we can show by examples that Theorem 1.1 will not be

Received June 26, 1997. Revision received May 18, 1998.

Research partially supported by NSF Grant no. DMS-9626310.

Michigan Math. J. 45 (1998). 
true if any of the assumptions is removed. We also should point out that, besides the curvature assumption, we only use the fact that the target manifold $N$ satisfies Sobolev inequality in the proof of Theorem 1.1. Hence Theorem 1.1 still holds for more general target manifolds-for example, when $N$ is a minimal submanifolds of $\mathbf{R}^{K}$ - since in that case we know from [MS] that Sobolev inequality holds on $N$.

On the other hand, it is well known that there is no nonconstant holomorphic map from a complete Kähler manifold $M$ into a complex Hermitian manifold $N$ if $M$ has nonnegative Ricci curvature and $N$ has holomorphic bisectional curvature bounded from above by a negative constant. This follows easily from the generalized Schwarz lemma (cf. [Y]). Using a modified argument of Theorem 1.1, we can generalize this result to quasi-conformal diffeomorphisms as follows.

THEOREM 1.2. Let $M^{n}$ be a complete Riemannian manifold with $\lim _{r \rightarrow \infty} V_{p}(r)=$ $o\left(r^{n}\right)$, and let $N^{n}$ be a complete Riemannian manifold with $\lambda_{1}(N)>0$, where $n$ is the dimension of both manifolds, $p \in M$ is any fixed point, $V_{p}(r)$ is the volume of the ball of radius $r$ centered at $p$, and $\lambda_{1}(N)$ is the lower bound of the spectrum of the Laplacian-Beltrami operator. Then there is no quasi-conformal diffeomorphism from $M$ into $N$.

We know that $\lambda_{1}(N)>0$ if $N$ is simply connected and has sectional curvature bounded from above by some negative constant. That is why we call Theorem 1.2 a generalization of the aforementioned result on holomorphic maps (which is derived from the generalized Schwarz lemma). The interesting thing is that Theorem 1.2 is invariant under the quasi-isometries whereas Theorem 1.1 is not.

AcKnowledgments. The author would like to thank his advisor, Prof. P. Li, for much valuable advice, as well as Prof. J. Wang for helpful suggestions. The author would also like to thank the referee for many valuable comments, which were of great help in improving the exposition of this paper.

\section{Proofs of the Theorems}

Proof of Theorem 1.1. We prove by contradiction.

Assume that there is a harmonic diffeomorphism $u$ from $M$ into $N$. Let $a^{2}(x)=$ $\inf _{\left\{v \in T M_{x} \mid\|v\|=1\right\}}\left|(d u)^{*} \circ(d u)(v)\right|^{2}$. By choosing a suitable orthonormal frame $\left\{e_{i}\right\}$ around $x$ in $T M$, we have that

$$
(d u)^{*} \circ(d u)=\left(\begin{array}{ccc}
\lambda_{1}^{2} & \ldots & 0 \\
\vdots & \vdots & \vdots \\
0 & \ldots & \lambda_{n}^{2}
\end{array}\right) .
$$

We can assume that $\lambda_{1}^{2} \geq \lambda_{2}^{2} \geq \cdots \geq \lambda_{n}^{2}=a^{2}(x)$. By definition we have $e(u)=$ $\sum_{i=1}^{n} \lambda_{i}^{2}$ and $J(u)=\lambda_{1} \cdots \lambda_{n}$.

Let $\phi(x)$ be a smooth function with compact support defined on $M$. Then $\phi \circ u^{-1}(y)$ is a smooth function with compact support defined on $N$. By the assumption on $N$ we know that the Sobolev inequality holds (cf. [HS]); that is, we have a constant $S$ such that 


$$
\int_{N}|\nabla \varphi| d v_{N} \geq S\left(\int_{N}|\varphi|^{n /(n-1)}\right)^{(n-1) / n} \quad \text { for all } \varphi \in C_{0}^{\infty}(N) .
$$

Applying this to $\phi \circ u^{-1}$ yields

$$
\int_{N}\left|\left(\nabla \phi \circ u^{-1}\right)(y)\right| d v_{N} \geq S\left(\int_{N}\left|\left(\phi \circ u^{-1}\right)(y)\right|^{n /(n-1)}\right)^{(n-1) / n} .
$$

On the other hand, direct calculation shows that

$$
\begin{aligned}
\int_{N}\left|\nabla_{N}\left(\phi \circ u^{-1}\right)(y)\right| d v_{N} & \leq \int_{M}\left|\left(\nabla_{M} \phi\right)(x)\right| a^{-1}(x) J(x) d v_{M} \\
& \leq \int_{M}\left|\nabla_{M} \phi\right|\left|\lambda_{1}(x) \cdots \lambda_{n-1}(x)\right| d v_{M} .
\end{aligned}
$$

Using the arithmetic-geometric inequality

$$
\left(\lambda_{1} \cdots \lambda_{n-1}\right)^{2 /(n-1)} \leq \frac{\sum_{i=1}^{n-1} \lambda_{i}^{2}(x)}{n-1} \leq \frac{e(u)}{n-1},
$$

we have

$$
\int_{M}\left|\nabla_{N}\left(\phi \circ u^{-1}\right)(y)\right| d v_{N} \leq C(n) \int_{M}\left|\left(\nabla_{M} \phi\right)(x)\right| e^{(n-1) / 2} d v_{M} .
$$

Combining (2.1) and (2.2) yields

$$
\left(\int_{N}\left|\left(\phi \circ u^{-1}\right)(y)\right|^{n /(n-1)}\right)^{(n-1) / n} \leq C(n, S) \int_{M}\left|\nabla_{M} \phi\right| e^{(n-1) / 2} d v_{M}
$$

Now we estimate $\left(\int_{N}\left|\left(\phi \circ u^{-1}\right)(y)\right|^{n /(n-1)}\right)^{(n-1) / n}$ using the quasi-conformity of $u$ as follows.

Recall the definition of the quasi-conformal constant to be $\alpha=\sup _{x \in M}\left(\lambda_{1} / \lambda_{n}\right)$. Then we have $\lambda_{1} / \lambda_{i} \leq \alpha$, and

$$
\begin{gathered}
J(x)=\lambda_{1} \cdots \lambda_{n} \geq \alpha^{1-n}(a(x))^{n} \\
e(x)=\sum_{i=1}^{n} \lambda_{i}^{2} \leq a(x)^{2}\left((n-1) \alpha^{2}+1\right) .
\end{gathered}
$$

Combining these yields

$$
\begin{aligned}
\left(\int_{N}\left|\left(\phi \circ u^{-1}\right)(y)\right|^{n /(n-1)}\right. & \left.d v_{N}\right)^{(n-1) / n} \\
& \geq C(n, \alpha)\left(\int_{M}|\phi(x)|^{n /(n-1)} e^{n / 2} d v_{M}\right)^{(n-1) / n} .
\end{aligned}
$$

Together with (2.3), inequality (2.4) implies

$$
\left(\int_{M}|\phi|^{n /(n-1)} e^{n / 2} d v_{M}\right)^{(n-1) / n} \leq C(n, \alpha, S) \int_{M}\left|\nabla_{M} \phi\right| e^{(n-1) / 2} d v_{M} .
$$

The rest of the proof is just deriving a contradiction from (2.5). 
Let $S(r)=\sup _{B_{p}(r)} e(u)$. It is well known that $e(u)$ is a subharmonic function under our curvature assumptions. By the mean value inequality for subharmonic functions on manifolds with nonnegative Ricci curvature, we have

$$
S\left(\frac{r}{2}\right) \leq C(n) \frac{1}{V_{p}(r)} \int_{B_{p}(r)} e(u)(x) d v_{M} .
$$

By choosing $\phi$ to be

$$
\begin{aligned}
& \phi(x)= \begin{cases}1 & \text { for } x \in B_{p}(r), \\
0 & \text { for } x \in M \backslash B_{p}(2 r),\end{cases} \\
& |\nabla \phi| \leq C / r \quad \text { with } C=2 \text {, }
\end{aligned}
$$

inequality (2.5) yields

$$
\begin{aligned}
& \left(\frac{1}{V_{p}(r)} \int_{B_{p}(r)} e(u)^{n / 2} d v_{M}\right)^{(n-1) / n} \\
& \quad \leq C(n, \alpha, S) \frac{\left(V_{p}(r)\right)^{1 / n}}{r} \frac{V_{p}(2 r)}{V_{p}(r)} \frac{1}{V_{p}(2 r)} \int_{B_{p}(2 r)} e(u)^{(n-1) / 2} d v_{M} .
\end{aligned}
$$

Combining this with Li and Schoen's mean value inequality and the volume doubling property of Ricci nonnegative manifolds, we have

$$
\left(S\left(\frac{r}{2}\right)\right)^{(n-1) / 2} \leq C \frac{\left(V_{p}(r)\right)^{1 / n}}{r}(S(2 r))^{(n-1) / 2} .
$$

Now we use the polynomial growth condition on the energy density of $u$. The polynomial growth assumption is simply that there exists a constant $K$ such that $S(r) \leq K\left(1+r^{d}\right)$ for some $d \geq 0$. But it is not hard to show that the polynomial growth condition implies the existence of a constant $A>0$ and $r_{j} \rightarrow \infty$ such that

$$
\frac{S\left(2 r_{j}\right)}{S\left(r_{j} / 2\right)} \leq A
$$

Applying (2.6) to $r_{j}$, we have

$$
\left(S\left(\frac{r_{j}}{2}\right)\right)^{(n-1) / 2}\left(1-C \frac{\left(V_{p}\left(r_{j}\right)\right)^{1 / n}}{r_{j}}(A)^{(n-1) / 2}\right) \leq 0 .
$$

Letting $r_{j} \rightarrow \infty$ and using that $V_{p}(r)=o\left(r^{n}\right)$, we have

$$
\lim _{r_{j} \rightarrow \infty} S\left(\frac{r_{j}}{2}\right) \leq 0 .
$$

Since $e(u)(x)$ is a subharmonic function and achieves its maximum at infinity, we have $e(u)(x) \equiv 0$, which is a contradiction because $u$ is a quasi-conformal diffeomorphism.

COROLlaRy 2.1. There is no quasi-conformal harmonic diffeomorphism from $\mathbf{S}^{k} \times \mathbf{R}^{n-k}$ into $\mathbf{R}^{n}$ with polynomial growth energy density. 
In order to prove Theorem 1.2 we need the following lemma, which is well known to the experts. For completeness we include a simple proof here.

LEMMA 2.2. If there exists a positive constant $A_{p}$ such that

$$
\int_{N}|\varphi|^{p} d v_{N} \leq A_{p} \int_{N}|\nabla \varphi|^{p} d v_{N} \quad \text { for any } \varphi \in C_{0}^{\infty}(N),
$$

then there exists a positive constant $A_{q}$ such that

$$
\int_{N}|g|^{q} d v_{N} \leq A_{q} \int_{N}|\nabla g|^{q} d v_{N} \quad \text { for any } g \in C_{0}^{\infty}(N),
$$

provided $q \geq p$. In other words, $L_{p}$-Poincaré implies $L_{q}$-Poincaré if $q \geq p$.

Proof. Let $\psi=|g|^{q / p}$. Then

$$
|\nabla \psi|=\frac{q}{p}|g|^{(q-p) / p}|\nabla g| .
$$

Applying (2.7) to $\psi$ yields

$$
\begin{aligned}
\int_{N}|g|^{q} d v_{N} & =\int_{N}|\psi|^{p} d v_{N} \\
& \leq A_{p} \int_{N}|\nabla \psi|^{p} d v_{N} \\
& =A_{p}\left(\frac{q}{p}\right)^{p} \int_{N}|\nabla g|^{p}|g|^{q-p} d v_{N} \\
& \leq A_{p}\left(\frac{q}{p}\right)^{p}\left(\int_{N}|\nabla g|^{q}\right)^{p / q}\left(\int_{N}|g|^{q}\right)^{(q-p) / q}
\end{aligned}
$$

Then we have (2.8) with $A_{q}=\left(A_{p}\right)^{q / p}(q / p)^{q}$.

Proof of Theorem 1.2. By the assumption on $N$, we now have the $L_{2}$-Poincaré inequality

$$
\lambda_{1} \int_{N}|\varphi|^{2} \leq \int_{N}|\nabla \varphi|^{2} \quad \text { for any } \varphi \in C_{0}^{\infty}(N) .
$$

Applying Lemma 2.2 yields

$$
\int_{N}|\varphi|^{n} d v_{N} \leq C\left(\lambda_{1}, n\right) \int_{N}|\nabla \varphi|^{n} d v_{n} .
$$

As in the proof of Theorem 1.1, we apply the preceding inequality to $\phi \circ u^{-1}$. Then we have

$$
\int_{N}\left|\left(\phi \circ u^{-1}\right)(y)\right|^{n} d v_{N} \leq C\left(\lambda_{1}, n\right) \int_{N}\left|\nabla_{N}\left(\phi \circ u^{-1}\right)(y)\right|^{n} d v_{N} .
$$

Similar calculation as in the proof of (2.2) shows

$$
\int_{N}\left|\nabla_{N}\left(\phi \circ u^{-1}\right)(y)\right|^{n} d v_{N} \leq C(\alpha) \int_{M}\left|\left(\nabla_{M} \phi\right)(x)\right|^{n} d v_{M} .
$$


On the other hand, the same calculation as in the proof of (2.4) shows that

$$
\int_{M}|\phi(x)|^{n} e(u)^{n / 2} d v_{M} \leq C(n, \alpha) \int_{N}\left|\left(\phi \circ u^{-1}\right)(y)\right|^{n} d v_{N} .
$$

Combing the preceding two inequalities and choosing $\phi$ as in the proof of Theorem 1.1, we will have

$$
\frac{V_{p}(r)}{r^{n}} \geq C\left(\lambda_{1}, \alpha, n\right) \int_{B_{p}(r)} e(u)^{n / 2} .
$$

Now our assumption on the volume growth yields $e(u) \equiv 0$, which completes the proof.

Combining the proofs of Theorem 1.1 and Theorem 1.2, we have the following corollary.

COROLlary 2.3. Let $M^{n}$ be a complete Riemannian manifold with nonnegative Ricci curvature, and let $N^{n}$ be a complete Riemannian manifold with nonpositive sectional curvature and $\lambda_{1}(N)>0$, where $n$ is the dimension of the both manifolds. Then there exists no quasi-conformal harmonic diffeomorphism from $M$ into $N$.

Finally, we present two examples. They will show that, in Theorem 1.1, both the volume growth assumption and the polynomial growth assumption of the energy density are indeed necessary.

ExAmple 1. This example shows that if we do not assume $V_{p}(r)=o\left(r^{n}\right)$ then we cannot have our theorem. Let $M=N=R^{n}$, and let $u$ be the identity map between the $R^{n}$. Then $u$ has bounded energy density and satisfies all the other assumptions of Theorem 1.1.

EXAMPLE 2. This example shows that if we do not have the growth condition on the energy density our theorem also fails. Here we let $M=S^{1} \times R$ and $N=R^{2}$, with $u$ a mapping from $M$ into $N$ (in fact onto $R^{2} \backslash\{0\}$ ) given by $\eta=e^{r}$ and $\omega=$ $\theta$, where $d r^{2}+d \theta^{2}$ is the metric on $M$ and $d \eta^{2}+\eta^{2} d \omega^{2}$ is the metric on $N$. We can see easily that $e(u)$ has exponential growth energy density and satisfies all the other assumptions of Theorem 1.1.

Added in proof. It turns out that the proof of Theorem 1.1 can be sharpened to prove the following stronger statement.

Let $M^{n}$ and $N^{n}$ be two complete Riemannian manifolds of same dimension. If there is a point $p \in M$ such that $\lim _{r \rightarrow \infty} V_{p}(r)=o\left(r^{n}\right)$ and Sobolev inequality holds on $N$, then there is no quasi-conformal diffeomorphism from $M$ into $N$ with polynomial growth energy density.

\section{References}

[C] S. Y. Cheng, Liouville theorem for harmonic maps, Proc. Sympos. Pure Math., 36, pp. 147-151, Amer. Math. Soc., Providence, RI, 1980. 
[HTTW] Z. Han, L. F. Tam, A. Treibergs, and T. Wan, Harmonic maps from the complex plane into surfaces with nonpositive curvature, Comm. Anal. Geom. 3 (1995), 85-114.

[HS] D. Hoffman and J. Spruck, Sobolev and isoperimetric inequalities for Riemannian submanifolds, Comm. Pure Appl. Math. 27 (1974), 715-727.

[LTW] P. Li, L. F. Tam, and J. Wang, Harmonic diffeomorphisms between Hadamard manifolds, Tran. Amer. Math. Soc. 347 (1995), 3645-3658.

[MS] J. Michael and L. M. Simon, Sobolev and mean-value inequalities on generalized submanifolds of $R^{n}$, Comm. Pure Appl. Math. 26 (1973), 361-379.

[S] R. Schoen, The role of harmonic mappings in rigidity and deformation problems, Collection: Complex geometry (Osaka, 1990), Lecture Notes in Pure and Appl. Math., 143, pp. 179-200, Dekker, New York, 1993.

[Sh] Y. Shen, A Liouville theorem for harmonic maps, Amer. J. Math. 117 (1995), 773-785.

[T] L. F. Tam, Liouville properties of harmonic maps, Math. Res. Lett. 2 (1995), 719-735.

[W] T. Wan, Constant mean curvature surfaces, harmonic maps, and universal Teichmüller space, J. Differential Geom. 35 (1992), 643-657.

[Y] S. T. Yau, A general Schwarz lemma for Kähler manifolds, Amer. J. Math. 100 (1978), 197-203.

Department of Mathematics

University of California - Irvine

Irvine, CA 92697

lni@math.uci.edu 